\title{
Functional Connectivity and Brain Networks in Schizophrenia
}

\author{
Mary-Ellen Lynall, ${ }^{1,2}$ Danielle S. Bassett, ${ }^{1,2,3,4}$ Robert Kerwin, ${ }^{\dagger}{ }^{\dagger}$ Peter J. McKenna, ${ }^{6}$ Manfred Kitzbichler, ${ }^{1,2}$ \\ Ulrich Muller, ${ }^{1,2}$ and Ed Bullmore ${ }^{1,2,7}$ \\ ${ }^{1}$ Behavioural and Clinical Neuroscience Institute and 2Department of Psychiatry, University of Cambridge, Cambridge CB2 0SZ, United Kingdom, \\ ${ }^{3}$ Cognition, and Psychosis Program, Clinical Brain Disorders Branch, Genes, National Institute of Mental Health, National Institutes of Health, Bethesda, \\ Maryland 20892, ${ }^{4}$ Biological Soft Systems Sector, Department of Physics, University of Cambridge, Cambridge CB3 0HE, United Kingdom, ${ }^{5}$ Institute of \\ Psychiatry, King's College London, London SE5 8AF, United Kingdom, ${ }^{6}$ Benito Menni Complex Assistencial en Salut Mental, Centro de Investigacion en \\ Red de Salut Mental, Sant Boi de Llobregat, Barcelona 08840, Spain, and ${ }^{7}$ Clinical Unit Cambridge, GlaxoSmithKline, Addenbrooke's Hospital, Cambridge \\ CB2 0QQ, United Kingdom
}

Schizophrenia has often been conceived as a disorder of connectivity between components of large-scale brain networks. We tested this hypothesis by measuring aspects of both functional connectivity and functional network topology derived from resting-state fMRI time series acquired at 72 cerebral regions over $17 \mathrm{~min}$ from 15 healthy volunteers ( 14 male, 1 female) and 12 people diagnosed with schizophrenia (10 male, 2 female). We investigated between-group differences in strength and diversity of functional connectivity in the $0.06-0.125 \mathrm{~Hz}$ frequency interval, and some topological properties of undirected graphs constructed from thresholded interregional correlation matrices. In people with schizophrenia, strength of functional connectivity was significantly decreased, whereas diversity of functional connections was increased. Topologically, functional brain networks had reduced clustering and small-worldness, reduced probability of high-degree hubs, and increased robustness in the schizophrenic group. Reduced degree and clustering were locally significant in medial parietal, premotor and cingulate, and right orbitofrontal cortical nodes of functional networks in schizophrenia. Functional connectivity and topological metrics were correlated with each other and with behavioral performance on a verbal fluency task. We conclude that people with schizophrenia tend to have a less strongly integrated, more diverse profile of brain functional connectivity, associated with a less hub-dominated configuration of complex brain functional networks. Alongside these behaviorally disadvantageous differences, however, brain networks in the schizophrenic group also showed a greater robustness to random attack, pointing to a possible benefit of the schizophrenia connectome, if less extremely expressed.

\section{Introduction}

It was first proposed by 19th century pioneers such as Theodor Meynert (1833-1892) and Carl Wernicke (1848-1905) that psychotic disorders might arise from abnormal axonal connectivity between anatomically dissected cortical regions. This seminal hypothesis of disconnection or cortical miswiring in psychosis (Catani and ffytche, 2005; Catani and Mesulam, 2008), based on morbid anatomy and clinical intuition, has more recently been gen-

\footnotetext{
Received Dec. 13, 2009; revised April 14, 2010; accepted May 19, 2010.

This research was supported by a Human Brain Project grant from the National Institute of Mental Health and the National Institute of Biomedical Imaging and Bioengineering. Data acquisition was supported by a grant from Bristol Myers Squibb. D.S.B. was supported by the National Institutes of Health-Cambridge Graduate Partnership Program P.J.M. was supported by the Instituto de Salud Carlos III, Centro de Investigación en Red de Salut Mental, CIBERSAM We thank Dr. Rebecca Jones for her contribution to patient recruitment, and Glyn Johnson for supervising fMR acquisition at the BUPA Lea Hospital (Cambridge, UK).

This paper is dedicated to the memory of Prof. Robert W. Kerwin (1955-2007), neuropharmacologist and psychiatrist.

${ }^{\dagger}$ Deceased, February 8, 2007.

E.B. is employed half-time by GlaxoSmithKline.

Correspondence should be addressed to Ed Bullmore, Department of Psychiatry, University of Cambridge, Herchel Smith Building for Brain and Mind Sciences, Cambridge Biomedical Campus, Cambridge CB2 0SZ, UK. E-mail: etb23@cam.ac.uk.

DOI:10.1523/JNEUROSCI.0333-10.2010

Copyright $\odot 2010$ the authors $\quad 0270-6474 / 10 / 309477-11 \$ 15.00 / 0$
}

eralized to the concept of dysconnectivity: abnormal relationships between neurons, at multiple scales of space and time, compatible with—but not necessarily implying-anatomical disconnection (Volkow et al., 1988; Weinberger et al., 1992; Friston and Frith, 1995; Friston, 1996; Bullmore et al., 1997). Dysconnectivity in schizophrenia is considered an intermediate disease phenotype, conceivably attributable to various degenerative, developmental, and/or genetic mechanisms (Meyer-Lindenberg and Weinberger, 2006). One distinctive and mechanistically plausible hypothesis links functional dysconnectivity at the macro-scale of neuroimaging to abnormal synaptic modulation at the micro-scale of cellular signaling (Stephan et al., 2009).

Meta-analytic reviews of MRI studies of schizophrenia have provided strong evidence for abnormal gray matter density increases in basal ganglia, and decreases in bilateral frontal, cingulate, temporal, and insular cortex, and thalamus (Ellison-Wright et al., 2008; Glahn et al., 2008). Diffusion tensor imaging (DTI) studies of white matter organization have replicably found reduced anisotropy of diffusion in left frontal and temporal lobes (Ellison-Wright and Bullmore, 2009). Convergent evidence across diverse cognitive task conditions also indicates abnormal fMRI activation of dorsal and ventral prefrontal, anterior cingu- 


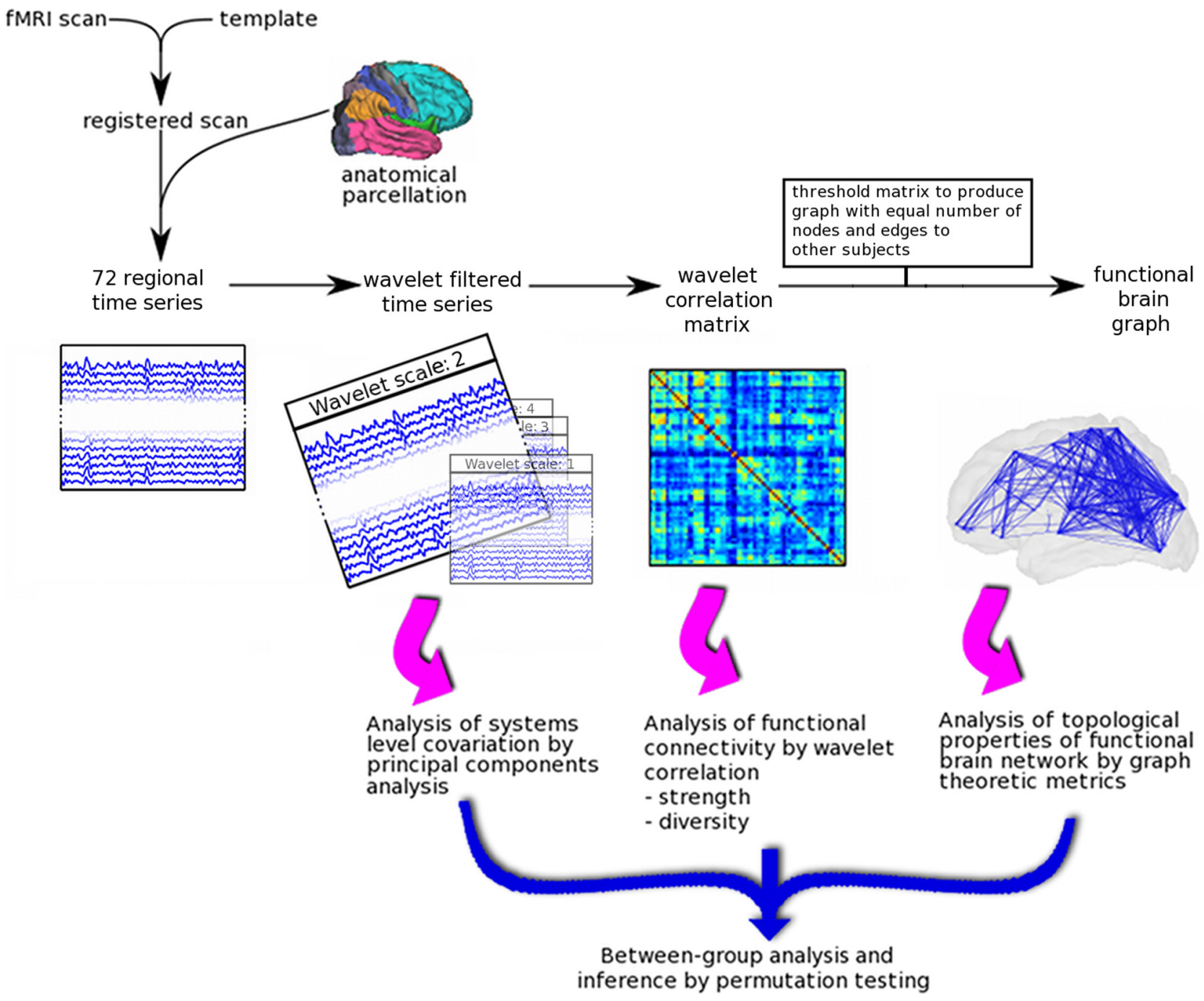

Figure 1. Schematic of fMRI data analysis pipeline. Regional mean fMRI time series were estimated by applying a prior anatomical template image to each individual fMRI dataset after its coregistration with the template in standard space; wavelet analysis was used to bandpass filter the regional time series and to estimate frequency-specific measures of functional connectivity between regions; functional connectivity matrices were thresholded to generate binary undirected graphs or brain functional networks; between-group differences in functional connectivity, principal components, and network topological metrics were assessed by permutation testing.

late, and posterior cortical regions (Minzenberg et al., 2009). The most parsimonious explanation of this pattern of multiple local structural and functional abnormalities is that schizophrenia is represented at the scale of neuroimaging by a disconnected configuration of these gray matter regions and their interconnecting white matter tracts in MRI and DTI data, which is somehow reflected in abnormal functional connectivity in fMRI data.

More direct support for the functional dysconnectivity hypothesis comes from resting-state fMRI studies of disorderrelated differences in interregional functional connectivity (Liang et al., 2006; Bluhm et al., 2007; Zhou et al., 2007a,b; Jafri et al., 2008; Whitfield-Gabrieli et al., 2009; Fornito and Bullmore, 2010; Salvador et al., 2010), defined as the statistical association between spatially distributed neurophysiological time series (Friston, 1994). In parallel, graph theoretic measurements of the topological properties of complex brain networks have found that they are less hierarchical, less small-world, less clustered, and less efficiently wired in schizophrenia (Bassett et al., 2008; Liu et al., 2008; Bullmore and Sporns, 2009). These differences might be expected to impair higher-order cognitive functions demanding access to large, integrated neuronal workspaces (Dehaene and
Naccache, 2001). Working memory impairments have been linked to reduced cost efficiency of magnetoencephalographic networks in schizophrenia (Bassett et al., 2009). However, if the functional consequences of altered topology in schizophrenia are entirely negative, why have evolutionary processes not selected against risk genes for this highly heritable disorder?

We measured functional connectivity and network metrics in no-task fMRI data recorded from 15 healthy volunteers and 12 people with schizophrenia, and we investigated how brain functional organization was expressed in terms of these various, interdependent metrics, and how it related to cognitive function; see Figure 1 for schematic overview.

\section{Materials and Methods}

Sample

We recruited 15 healthy (nonpsychotic) volunteers (14 male, 1 female) and 12 people with chronic schizophrenia (10 male, 2 female), diagnosed according to standard operational criteria in the Diagnostic and Statistical Manual of Mental Disorders IV (American Psychiatric Association, 2000). The two groups were matched for age, premorbid IQ estimated using the National Adult Reading Test (Nelson, 1992), and years of ed- 
Table 1. Demographic and clinical characteristics of the sample

\begin{tabular}{lll}
\hline & $\begin{array}{l}\text { Healthy volunteers } \\
(N=15) \text { (mean } \pm \text { SD) }\end{array}$ & $\begin{array}{l}\text { People with schizophrenia } \\
(N=12) \text { (mean } \pm \text { SD) }\end{array}$ \\
\hline Age (years) & $33.3 \pm 9.2$ & $32.8 \pm 9.2$ \\
Premorbid NART IQ & $113 \pm 6$ & $112 \pm 9$ \\
Years of education & $13.3 \pm 6.4$ & $12.8 \pm 2.4$ \\
Gender & 14 male, 1 female & 10 male, 2 female \\
Symptom severity (PANSS scale) & - & Positive: $4.0 \pm 4.2$ \\
& & Negative: $8.3 \pm 5.4$ \\
& & General: $15.4 \pm 9.8$ \\
\hline
\end{tabular}

NART, National Adult Reading Test.

ucation. Symptom severity scores were measured using the Positive and Negative Syndrome Scale (PANSS) scale (Kay et al., 1987). For subject details, see Table 1 . All patients were receiving antipsychotic drugs, and four were receiving additional psychotropic medication. The average $( \pm$ SD) dose, in chlorpromazine equivalents (Woods, 2003; Bazire, 2005), was $487 \pm 433 \mathrm{mg} / \mathrm{d}$. To mitigate acute drug effects on fMRI data, patients did not receive their usual medication on the day of scanning. Healthy volunteers were screened for major psychiatric disorders using the Mini International Neuropsychiatric Interview (Sheehan et al., 1998); none were taking psychoactive medication. All subjects provided informed consent in writing and the protocol was approved by the Addenbrooke's NHS Trust Local Research Ethics Committee.

\section{Cognitive testing}

The FAS version of the Controlled Oral Word Association Test (Benton et al., 1976) was used to assess verbal fluency. The participant was asked to say as many words as possible beginning with the letter F, A, or S within $1 \mathrm{~min}$, and the test score was simply the total number of words generated. Forward and backward digit span was assessed by Wechsler Memory Scale-III Digit Span (Wechsler, 1997).

\section{Acquisition and preprocessing of $f M R I$ data}

Functional MRI data were acquired while subjects were lying quietly in the scanner with eyes closed for $17 \mathrm{~min} 12 \mathrm{~s}$. We used a GE Signa system (General Electric) operating at $1.5 \mathrm{~T}$ at the BUPA Lea Hospital (Cambridge, UK). In each session, 516 gradient-echo T2*-weighted echo planar images depicting blood oxygenation level-dependent contrast were acquired from 16 noncontiguous near-axial planes: repetition time $=2 \mathrm{~s}$, echo time $=40 \mathrm{~ms}$, flip angle $=70^{\circ}$, voxel size $=3.05 \times 3.05 \times 7.00 \mathrm{~mm}$, section skip $=0.7 \mathrm{~mm}$, matrix size $=64 \times 64$, field of view $(\mathrm{FOV})=$ $240 \times 240 \times 123 \mathrm{~mm}$. The first 4 volumes were discarded to allow for T1 equilibration effects, leaving 512 volumes per session. Each dataset was corrected for head movement by realignment and regression (Suckling et al., 2006) and subsequently registered to MNI stereotactic standard space by a 12 parameter affine transform maximizing normalized correlation with a customized EPI template image (within-modality). Registered images were spatially smoothed with a Gaussian kernel $(6 \mathrm{~mm}$ at full width half-maximum), and the time series were high-pass filtered (cutoff frequency: $1 / 120 \approx 0.008 \mathrm{~Hz}$ ).

\section{Anatomical parcellation and wavelet decomposition}

For each individual dataset, up to 90 regional mean time series were estimated by averaging voxel time series within each of the 90 anatomically defined regions (excluding the cerebellum) comprising the Automated Anatomical Labeling (AAL) template image (Tzourio-Mazoyer et al., 2002). Because of the limited FOV size in the $z$-dimension, cerebellar regions had to be omitted to ensure sufficient coverage at the top of the brain. Regional time series were only included in further analysis if good quality fMRI data were available for $>50 \%$ of subjects; due to susceptibility artifacts at the base of the brain, this criterion excluded 18 regions from consideration (supplemental Table 1, available at www.jneurosci. org as supplemental material), leaving a complete dataset of 72 regions (supplemental Table 2, available at www.jneurosci.org as supplemental material) for all participants.

The maximal overlap discrete wavelet transform (Percival and Walden, 2000) was used to decompose each individual regional mean fMRI time
Table 2. Global functional connectivity measures and associated group differences at different frequency intervals

\begin{tabular}{|c|c|c|c|c|c|}
\hline \multirow[b]{2}{*}{ Frequency band $(\mathrm{Hz})$} & \multirow[b]{2}{*}{ Healthy } & \multirow[b]{2}{*}{ Schizophrenia } & \multicolumn{2}{|c|}{$t$ test $(\mathrm{df}=25)$} & \multirow{2}{*}{$\begin{array}{l}\text { Permutation } \\
\text { test }(p)\end{array}$} \\
\hline & & & $p$ & $t$ & \\
\hline \multicolumn{6}{|l|}{ Wavelet correlation $^{a}$} \\
\hline $0.125-0.250$ & 0.3213 & 0.2752 & 0.1899 & 1.3473 & 0.1045 \\
\hline $0.060-0.125$ & 0.4238 & 0.3289 & 0.0123 & 2.6974 & 0.0070 \\
\hline $0.030-0.060$ & 0.5063 & 0.4382 & 0.1235 & 1.5938 & 0.0610 \\
\hline $0.015-0.030$ & 0.5806 & 0.5119 & 0.1761 & 1.3924 & 0.0905 \\
\hline \multicolumn{6}{|c|}{ Wavelet mutual information ${ }^{b}$} \\
\hline $0.125-0.250$ & 0.0383 & 0.0344 & 0.3812 & 0.8914 & 0.2130 \\
\hline $0.060-0.125$ & 0.0528 & 0.0407 & 0.0305 & 2.2933 & 0.0130 \\
\hline $0.030-0.060$ & 0.0720 & 0.0625 & 0.3065 & 1.0440 & 0.1580 \\
\hline $0.015-0.030$ & 0.0983 & 0.0849 & 0.2543 & 1.1668 & 0.1345 \\
\hline
\end{tabular}

Bold indicates significance.

${ }^{a}$ Repeated-measures ANOVA: group effect, $F=3.944, p=0.0581$, df $=1$; frequency band effect, $F=81.15$, $p<0.0001, \mathrm{df}=3 ;$ group $\times$ frequency band interaction, $F=0.6754, p=0.5704, \mathrm{df}=3$.

${ }^{b}$ Repeated-measures ANOVA: group effect, $F=2.229, p=0.1479, \mathrm{df}=1$; frequency band effect, $F=79.83$, $p<0.0001, \mathrm{df}=3$; group $\times$ frequency band interaction, $F=0.5857, p=0.6262, \mathrm{df}=3$.

series into the following scales or frequency intervals: scale 1, 0.125$0.250 \mathrm{~Hz}$; scale 2, $0.060-0.125 \mathrm{~Hz}$; scale $3,0.030-0.060 \mathrm{~Hz}$; and scale 4, $0.015-0.030 \mathrm{~Hz}$. Following initial analyses of functional connectivity at all scales (Table 2), subsequent analysis focused on data at scale 2, which is compatible with prior studies indicating that endogenous fMRI dynamics of neuronal origin are most salient at frequencies of $<0.1 \mathrm{~Hz}$.

\section{Functional connectivity metrics}

At each scale, the wavelet correlation, $-1 \leq r_{i, j} \leq+1$, and mutual information, $m_{i, j} \geq 0$, were estimated between each possible $\{i, j\}$ pair of regions. Although wavelet correlations can be negative in these (and other) fMRI data, we have found that they are almost always positive (supplemental Fig. 1, available at www.jneurosci.org as supplemental material). This is an indication that connections between regions are not predominantly conferred through anti-correlation, which we would have had to treat separately otherwise. Connectivity strength, $\bar{R}$, and average mutual information, $\bar{M}$, were defined for each subject as the mean of all pairwise correlations or mutual informations, respectively.

Connectivity strength is a global measure of connectivity. The regional strength of connectivity $\bar{R}(i)$ was likewise defined for the $i$ th region as the average of the correlations between it and all other regions in the brain:

$$
\bar{R}(i)=\frac{\sum_{j} r_{i, j}}{N-1} .
$$

The regional diversity of connections, $\operatorname{Var}(R(i))$, was defined as the variance of the correlations between the $i$ th index region and all other regions:

$$
\operatorname{Var}(R(i))=\frac{\sum_{j}\left(r_{i, j}-\bar{R}(i)\right)^{2}}{N-1} .
$$

Globally, connectivity diversity was defined as the average regional diversity across the 72 brain regions.

Principal component analysis (PCA) was performed on the scale 2 wavelet coefficients, and a measure of global integration (Tononi et al., 1994; Friston, 1996) was estimated by the ratio of the first eigenvalue to the sum of all other eigenvalues: $I=\left(\lambda_{1}\right) / \sum_{j=2}^{N} \lambda_{j}$.

\section{Functional network metrics}

Undirected graphs were constructed from the scale 2 wavelet correlation matrices (Achard et al., 2006; Achard and Bullmore, 2007; Meunier et al., 2009) [see Bullmore and Sporns (2009) for a general review of graph theory in relation to neuroscience]. Any correlation $r_{i, j}$ in the functional connectivity matrix $\mathbf{C}$ greater than a given threshold, $\tau$, was retained as an edge connecting regions $i$ and $j$ in the adjacency matrix $\mathbf{A}$; if $r_{i, j}<\tau$, no edge connects regions $i$ and $j$. Graphs of different connection densities or costs are produced by thresholding at different values of $\tau$; the connection density is the number of edges in a graph comprising $N$ nodes 
divided by the maximum number of possible edges $\left[\left(N^{2}-N\right) / 2\right]$. When studying the topological properties of such graphs across a number of individuals, we prefer to consider graphs that are fully connected for all subjects (i.e., degree $k(i)>1$ for all nodes) and that have nonrandom topological organizational properties. These criteria defined a regime of cost or connection densities in the range $37-50 \%$ : below a connection density of $37 \%$, some graphs began to fragment, and above a connection density of $50 \%$, graph topology becomes increasingly random (Humphries et al., 2006) and less small-world. Work on brain connectivity in macaques (Kaiser and Hilgetag, 2006) suggests that connections at higher costs are likely to be nonbiological. All network results reported in this study are thus averages of the various metrics estimated for each individual network over a range of connection densities 37-50\% (14 values, $1 \%$ increments) (Bassett et al., 2008). The following graph metrics were estimated.

Degree. Degree, $k(i)$, is simply equal to the number of edges connecting the $i$ th region to the rest of the network:

$$
k(i)=\sum_{j} A_{i, j},
$$

where $\mathbf{A}$ is the binary adjacency matrix obtained by thresholding the functional connectivity matrix, $\mathbf{C}$.

Regional efficiency. Regional efficiency, E(i) (Latora and Marchiori, 2001; Achard and Bullmore, 2007), is computed for each node in a graph, $G$, as follows:

$$
E(i)=\frac{1}{N-1} \sum_{j \in G} \frac{1}{L_{i, j}} .
$$

Here $L_{i, j}$ is the minimum path length between regions $i$ and $j$. Global efficiency, $E(G)$, is the mean regional efficiency over all nodes.

Clustering coefficient. Clustering coefficient, $C(i)$, of a node $v$ is the ratio of connected triangles, $\delta_{v}$, to connected triples, $\tau_{v}$. The clustering coefficient of a graph is as follows:

$$
C(G)=\frac{1}{\left|V^{\prime}\right|} \sum_{\nu \in V^{\prime}} \frac{\delta_{\nu}}{\tau_{\nu}},
$$

where $V^{\prime}$ is the set of nodes with degree $>2$ (Watts and Strogatz, 1998; Schank and Wagner, 2005).

Small-worldness. Small-worldness, $\sigma$, is a property of a network with high clustering, $C$, but low characteristic path length, $L$, compared to the clustering, $C_{\mathrm{R}}$, and path length, $L_{\mathrm{R}}$, of a comparable random graph (Watts and Strogatz, 1998; Humphries et al., 2006). Path length can be estimated as the inverse of global efficiency (Latora and Marchiori, 2001), allowing the following formulation (Achard and Bullmore, 2007) of small-worldness:

$$
\sigma=\frac{C / C_{\mathrm{R}}}{E(G)_{\mathrm{R}} / E(G)}
$$

where $E(G)_{\mathrm{R}}$ is the global efficiency of a comparable random graph. A network is said to be "small-world" when $\sigma>1$.

Robustness. Robustness, $\rho$, indicates the network's resilience to either targeted, $\rho_{\mathrm{t}}$, or random, $\rho_{\mathrm{r}}$, attack. In a targeted attack, hubs are removed one by one in order of degree, $k$, while in a random attack, nodes are removed at random independent of their degree. Each time a node was removed from the network, we recalculated the size of the largest connected component, $s$. Robustness is then usually visualized by a plot of the size of the largest connected component, $s$, versus the number of nodes removed, $n$ (Achard et al., 2006) (supplemental Fig. 2, available at www.jneurosci.org as supplemental material). The robustness parameter, $\rho$, is defined as the area under this $s$ versus $n$ curve. More robust networks retain a larger connected component even when several nodes have been knocked out, as represented by a larger area under the curve or higher values of $\rho$.

Degree distribution parameters. Degree distribution parameters for graphs at a cost of $37 \%$ were estimated using the nonlinear fitting function in "Brainwaver" software [http://cran.r-project.org/ (Achard, 2007)]. For each subject, goodness of fit of the degree distribution to
Table 3. Functional connectivity and network topology metrics in the frequency interval $0.06-0.125 \mathrm{~Hz}$ for healthy volunteers and people with schizophrenia

\begin{tabular}{lccc}
\hline & $\begin{array}{l}\text { Healthy } \\
\text { volunteers } \\
\text { (mean } \pm \text { SD) }\end{array}$ & $\begin{array}{l}\text { People with } \\
\text { schizophrenia } \\
\text { (mean } \pm \text { SD) }\end{array}$ & $\begin{array}{l}p \text { value, } \\
\text { permutation } \\
\text { test }\end{array}$ \\
\hline Connectivity strength & $0.4238 \pm 0.0811$ & $0.3289 \pm 0.1018$ & 0.007 \\
Connectivity diversity & $0.0240 \pm 0.0047$ & $0.0282 \pm 0.0046$ & 0.016 \\
Variance of 1st PC & $43.1 \pm 8.4 \%$ & $32.6 \pm 11 \%$ & 0.005 \\
Global efficiency & $0.7439 \pm 0.0044$ & $0.7475 \pm 0.0030$ & 0.009 \\
Average clustering & $0.7423 \pm 0.0364$ & $0.6917 \pm 0.0562$ & 0.005 \\
Hierarchy & $0.0371 \pm 0.0086$ & $0.1013 \pm 0.0107$ & 0.010 \\
Degree distr. (variance) & $183 \pm 38$ & $120 \pm 43$ & $<0.0001$ \\
Degree distr. (power exponent) & $3.259 \pm 1.919$ & $6.116 \pm 3.427$ & 0.005 \\
Degree distr. (degree cut-off) & $9.450 \pm 2.896$ & $5.373 \pm 2.383$ & 0.0005 \\
Small-worldness & $1.6144 \pm 0.0745$ & $1.5300 \pm 0.1184$ & 0.015 \\
Robustness (random attack) & $2.534 \times 10^{3} \pm 11$ & $2.544 \times 10^{3} \pm 5$ & 0.001 \\
Robustness (targeted attack) & $2.454 \times 10^{3} \pm 44$ & $2.480 \times 10^{3} \pm 39$ & 0.065 \\
Verbal fluency & $15.27 \pm 3.86$ & $13.25 \pm 5.26$ & 0.1065
\end{tabular}

$p$ values refer to the probability of the observed between-group difference under the null hypothesis estimated by a permutation test. distr., Distribution.

three laws (exponential, $P(k) \sim e^{-\alpha k}$; power, $P(k) \sim k^{-\alpha}$; and truncated power, $\left.P(k) \sim k^{\alpha-1} e^{k / k_{c}}\right)$ was estimated using Akaike's information criterion. The exponentially truncated power law was the best fit for all subjects and the parameters of this distribution (the power exponent, $\alpha$, and the lower exponential degree cutoff, $k_{\mathrm{c}}$ ) were estimated for each subject.

\section{Correlations between variables}

We explored associations between all the functional connectivity, PCAbased, and graph theoretical metrics considered in the analysis of fMRI data (12 in total) (Table 3), simply using Pearson's correlation coefficient to estimate the association between each pair of variables over all subjects in the study $(N=27)$ (see Fig. 5). In general, all the fMRI metrics were (positively or negatively) correlated with each other (see supplemental Table 3, available at www.jneurosci.org as supplemental material, for details). As reported in more detail below, many of the brain functional metrics were also significantly correlated with behavioral variability in terms of verbal fluency scores. In an effort to isolate more specific associations between behavioral variability and brain functional metrics, we also estimated the partial correlations between each pair of variables and tested each of them for significance. We found that partial correlations were generally small and not significant, indicating that we cannot disambiguate any specific associations between behavioral variability and any one of the highly intercorrelated connectivity, PCA, or graph metrics considered in analysis of the fMRI data. We also used multivariate analysis of covariance to estimate the effects of all functional connectivity and network metrics on the dependent variable of verbal fluency. This analysis also demonstrated that no single metric demonstrated a specific relationship with cognitive performance when the effects of all other connectivity and network metrics were simultaneously considered. These results are reported in full in supplemental Tables 4 and 5 (available at www.jneurosci.org as supplemental material).

\section{Cortical surface rendering}

Caret v5.61 software (Van Essen et al., 2001) [with Atlas map (Van Essen, 2005)] was used to make cortical surface representations of the distributions of regional strength, regional diversity, degree, and clustering. The value plotted at a given point is the value of the AAL volume at a point below the surface at the level of cortical layer 4 . We tested the significance of the group differences in these metrics at each region using two-sample $t$ tests with a false-positive correction $p<(1 / N)=0.014$, which is equivalent to saying that we expect less than one false-positive regional result per cortical map at this threshold. We note that this correction for multiple comparisons is not as conservative as a Bonferroni or false discovery rate correction, and therefore we do not claim strong type I error control for these multiple exploratory analyses at a regional level of network organization. 
A Healthy volunteers
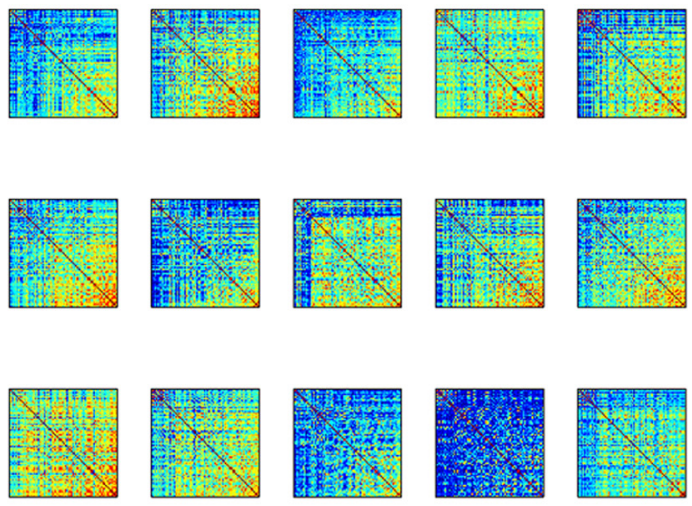

C

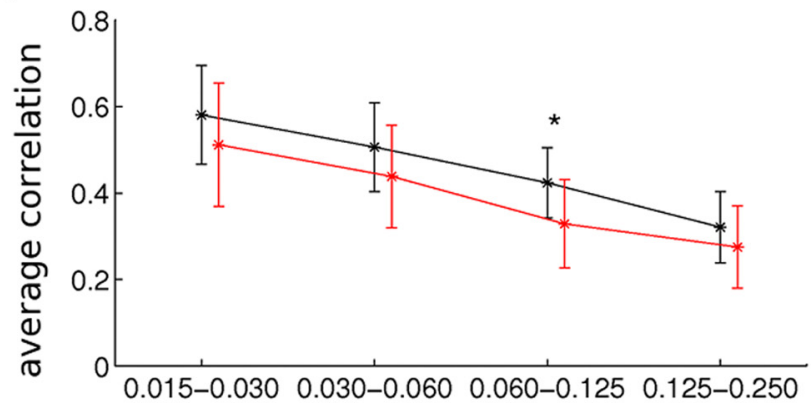

wavelet scale $(\mathrm{Hz})$
B People with schizophrenia
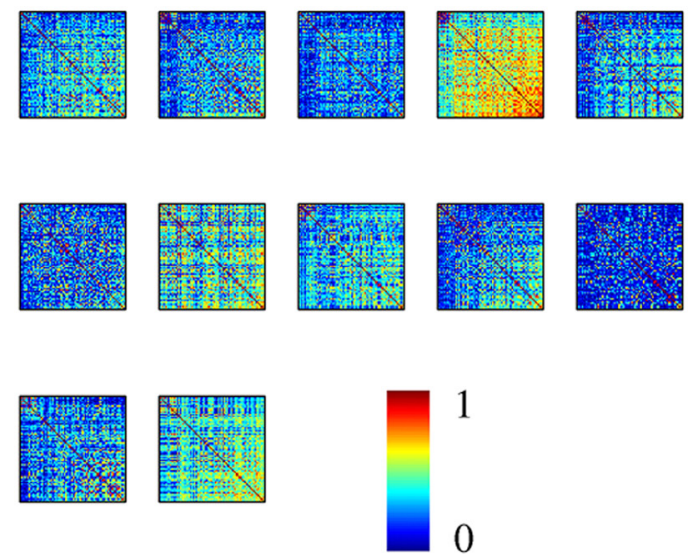

D

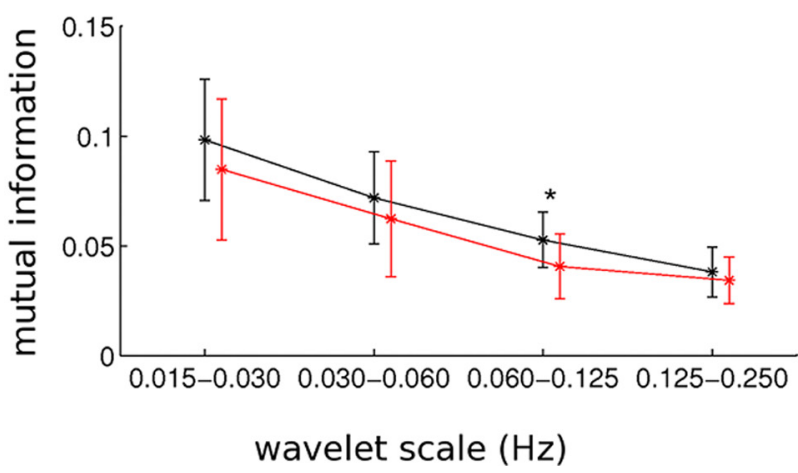

Figure 2. Functional connectivity matrices and group differences in global connectivity. $\boldsymbol{A}, \boldsymbol{B}$, Matrices of pairwise correlations at $0.060-0.125 \mathrm{~Hz}$ for individual participants: healthy controls $(\boldsymbol{A})$ and people with schizophrenia $(\boldsymbol{B})$. Both axes represent the 72 regions used in the analysis, ordered by average strength in healthy subjects, and pixel color represents the level of correlation. $\boldsymbol{C}$, $\boldsymbol{D}$, Connectivity strength, $\bar{R}(\boldsymbol{C}$ ), and average mutual information, $\bar{M}(\boldsymbol{D})$, at four different wavelet scales for healthy volunteers (black) and people with schizophrenia (red). The group differences denoted by asterisks were significant at the wavelet scale $0.060-0.125 \mathrm{~Hz}$ for connectivity strength and mutual information (Table 2). Error bars indicate SD.

\section{Results \\ Functional connectivity: strength, diversity, and global integration}

We measured the statistical association between spatially distributed pairs of regional fMRI time series using two metrics of frequency-specific functional connectivity. The wavelet correlation is a measure of the linear association between processes in a wavelet scale-specific frequency interval; the wavelet mutual information is a scale-specific measure of linear and nonlinear dependencies between processes. By both metrics, we found that the magnitude or strength of functional connectivity was greater at lower frequencies (Table 2, Fig. 2). This trend for bivariate correlations to be greater at lower frequencies is typical of the broad class of multivariate long memory time series models and is linked to the colored noise or persistent autocorrelation structure of a single fMRI time series (Achard et al., 2008). As anticipated by previous studies on resting-state networks in fMRI (Achard et al., 2006), the difference between schizophrenic and comparison groups was most salient by both metrics in the frequency interval $0.06-0.125 \mathrm{~Hz}$ (Table 2). Subsequent analysis focused in more detail on functional connectivity and networks based on the wavelet correlation matrices at this scale.

For each of 72 anatomically defined brain regions, we estimated the strength and diversity [or variability (Campbell et al., 1986)] of its functional connectivity to the rest of the brain in each individual dataset. Functional connectivity strength was generally greater, and ranged more widely over different brain regions, in healthy volunteers than in people with schizophrenia (Table 3, Fig. 3A). Connectivity strength was significantly reduced in the schizophrenic group at a regional level in medial premotor, cingulate and parietal cortex, precentral and postcentral cortex, occipital association cortex, and left inferior frontal, superior temporal, and insular cortex (Fig. 3C; supplemental Table 6, available at www.jneurosci.org as supplemental material). In contrast, the diversity of functional connections was significantly increased, on average over all regions, in the schizophrenic group (Table 3, Fig. 3B; supplemental Table 7, available at www. jneurosci.org as supplemental material). This difference was also significant at a regional level in orbitofrontal, insular, and parietal association cortex (Fig. 3D; supplemental Table 6, available at www.jneurosci.org as supplemental material). These two aspects of regional connectivity were negatively correlated over all subjects $(r=-0.4, \mathrm{df}=25, p=0.04)$ (Fig. 3E, see Fig. 5; supplemental Table 3, available at www.jneurosci.org as supplemental material). In other words, greater strength of connectivity was associated with reduced diversity of functional connections.

Using principal component (PC) analysis to provide a measure of the global integration of functional activity in each dataset, we found that the percentage of variance accounted for by the first PC was significantly reduced in people with schizophrenia (33\%) compared to healthy volunteers (43\%) (Fig. 3F, Table 3).

Taken overall, these results indicate that strength of brain functional connectivity is reduced, and that individual regions 
have a more diverse or less globally coordinated mode of connectivity to the rest of the brain, in people with schizophrenia.

Functional networks: topology, degree distributions, and robustness

To complement these results based on analysis of continuous measures of association between regions, we also measured the topological properties of binary (unweighted and undirected) graphs derived by thresholding the individual functional connectivity matrices.

At a global level, functional networks expressed some key organizational properties consistently across both groups. All individual networks had economical smallworld properties, i.e., high local and global efficiency, and broad scale degree distributions consistent with the existence of "hubs." However, the quantitative values of many of these topological metrics were significantly different between groups (Table 3, Fig. 4).

Clustering and small-worldness were significantly reduced by $\sim 5 \%$, and global efficiency was significantly increased by $<1 \%$, in the schizophrenic group. It was also notable that although the degree distributions of both groups were broadly similar, there were visible differences between them (Fig. 4A,B): both higherdegree hubs and lower-degree nodes were more probable in the healthy brain networks, whereas a greater proportion of nodes had modal degree in the schizophrenic brain networks. This was reflected by significantly higher brain-wide variance of regional degree in healthy volunteers (Table 3).

At a regional level of analysis, we mapped clustering and degree for each cortical node of the network and compared nodal clustering and degree between groups (Fig. 4). Consistent with the between-group differences in global topology, clustering was reduced for most cortical nodes in the schizophrenic group, although this difference was only significant for medial posterior parietal and anterior cingulate regions. Degree was also significantly reduced in medial posterior parietal and premotor cortex, and significantly increased in right orbitofrontal cortex, in the schizophrenic group (supplemental Table 8, available at www.jneurosci.org as supplemental material).

Possible forms of the degree distribution were evaluated more rigorously using Akaike's information criterion as a measure of comparative goodness of fit for three possible degree distributions: a power law, $P(k) \sim k^{-\alpha}$; an exponential, $P(k) \sim e^{-\alpha k}$; and an exponentially truncated power law, $P(k) \sim k^{\alpha-1} e^{k / k_{c}}$. Of these, the exponentially truncated power law was the best-fitting model for the degree distribution for all subjects in both groups. We compared the parameters of this distribution between the two groups (Table 3) and found that the exponential cutoff degree $\left(k_{\mathrm{c}}\right)$ was significantly lower in the schizophrenic group. This indicates that the transition from

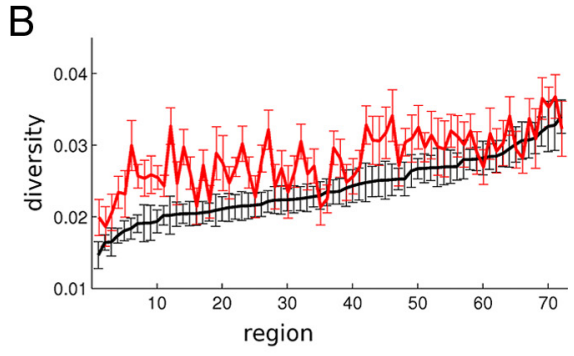

D
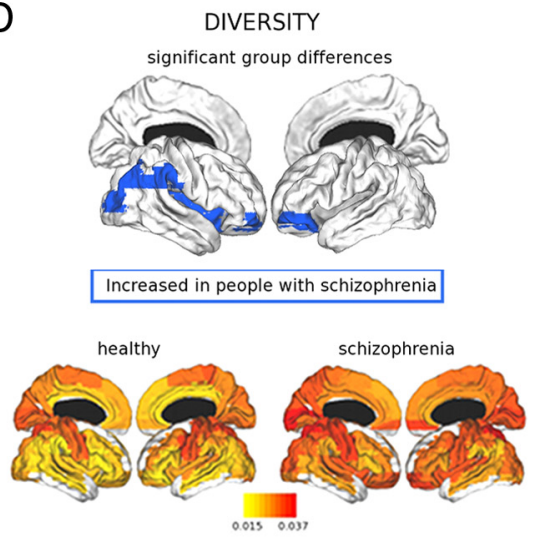

$\mathrm{F}$

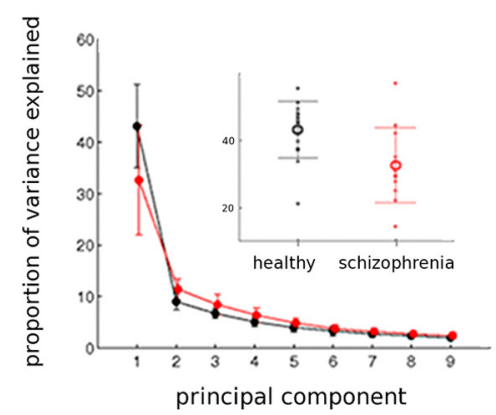

Figure 3. Group differences in regional connectivity metrics and global integration. $\boldsymbol{A}$, Group mean connectivity strength for each of the 72 regions, ordered by mean regional strength in healthy volunteers; error bars indicate $S E M$. $\boldsymbol{B}$, Regional diversity of relations, ordered by mean diversity in healthy volunteers; error bars indicate SEM. C, Cortical surface renderings of strength. comparisons using false-positive correction $(p<0.014$ ) are indicated. $\boldsymbol{E}$, Graph to show link between group differences in

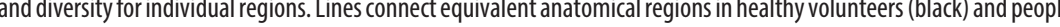
principal components in people with schizophrenia (red) and healthy volunteers (black). Inset shows the group difference in the proportion of variance explained by the first principal component. Error bars indicate SD. For details, see Table 3 and supplemental Tables 6 and 7 (available at www.jneurosci.org as supplemental material).

the scaling regimen to the exponential fall-off occurred at a lower degree in the schizophrenic group, which corresponds to a relative loss of hubs. We also found that the power exponent $(\alpha)$ was significantly higher in the schizophrenic group. Together with the lower exponential cutoff degree, this reflects the narrower degree distribution in the schizophrenic group.

We also investigated the robustness of the networks to random error (removal of nodes in random order) and targeted attack (removal of nodes in descending order of degree). Under both conditions, schizophrenic networks demonstrated greater robustness, and this was statistically significant for robustness to random error (Table 3; supplemental Fig. 1, available at www. jneurosci.org as supplemental material). Robustness was significantly negatively correlated with connectivity strength, global integration, and degree distribution parameters (Fig. 5; supplemental Table 3, available at www.jneurosci.org as supplemental material). 
A

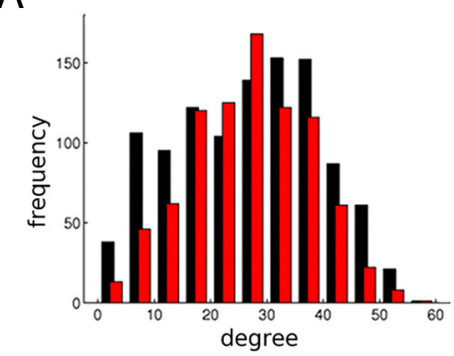

C
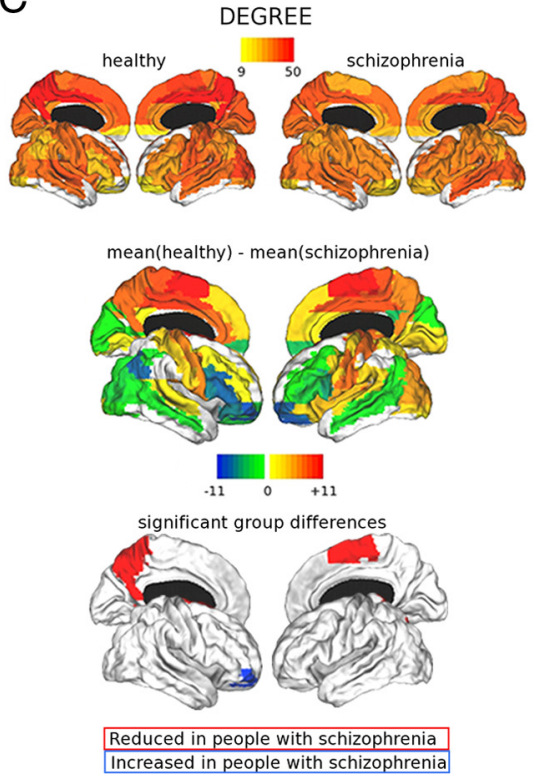

Increased in people with schizophrenia
$\mathrm{B}$

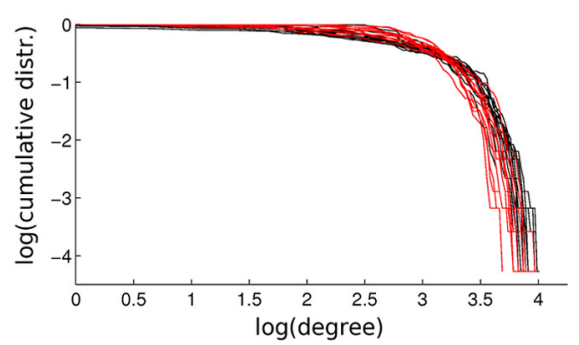

D
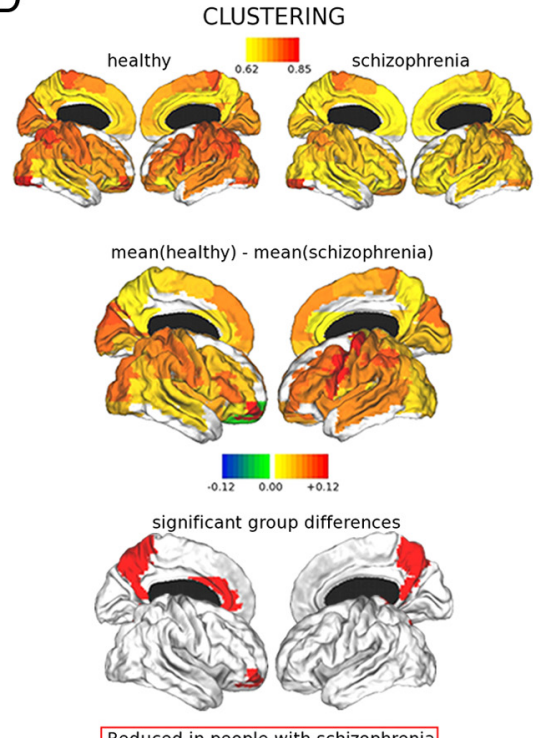

Reduced in people with schizophrenia

Figure 4. Group differences in topological properties of brain functional networks. $\boldsymbol{A}, \boldsymbol{B}$, Pooled degree distributions $(\boldsymbol{A})$ and cumulative degree distributions (B) for healthy volunteers (black) and people with schizophrenia (red), showing lower probability of high-degree network hubs in schizophrenia. C, D, Cortical surface renderings of degree (C) and clustering (D). Regions showing a significant group difference in the metric when corrected for multiple comparisons using false-positive correction $(p<0.014)$ are indicated. For details, see Table 3 and supplemental Table 8 (available at www.jneurosci.org as supplemental material).

\section{Relationships between functional connectivity, functional networks, and behavior}

Functional connectivity and network topology metrics were generally highly correlated (Fig. 5; supplemental Table 3, available at www.jneurosci.org as supplemental material). For example, greater strength and global integration of functional connectivity were positively correlated with greater small-worldness, greater clustering, and changes in degree distribution parameters indicating a higher probability of high-degree hubs.

Moreover, both connectivity and topological metrics were related to variability of behavioral performance on a test of verbal fluency. Greater fluency was positively correlated with greater connectivity strength and integration, greater small-worldness and clustering, and a more hub-dominated degree distribution. These associations were statistically significant when the correlations were tested pooling data from both groups (Fig. 5; supplemental Table 3, available at www.jneurosci.org as supplemental material), and the pattern of results was conserved when testing each group separately, although many of the within-group correlations were not statistically significant due to smaller sample size. Partial correlations, which estimate the component of covariation specifically attributable to the direct interaction between each pair of variables, were generally smaller than Pearson's correlations, and not statistically significant (see supplemental Table 4, available at www.jneurosci.org as supplemental material, for details).
There were no significant associations between any connectivity or topological metrics and either forward or backward digit span scores. There were also no significant associations, within the patient group, between clinical symptom severity [measured using the PANSS scale (Kay et al., 1987)] or current dose of atypical antipsychotic medication, and any of the brain functional measures.

\section{Discussion}

These results corroborate and extend prior studies indicating that brain systems measured by resting-state fMRI are abnormally organized in schizophrenia, as anticipated by theories of schizophrenia as a functional dysconnectivity syndrome.

\section{Functional connectivity and networks in schizophrenia}

One novel aspect of the study is that it is the first, we believe, to report a pathophysiological profile for schizophrenia in terms of both connectivity and topological metrics. Given that the topological metrics are estimated on a binary adjacency matrix constructed by thresholding the continuous association matrix of interregional connectivity measures, one would expect these two sets of metrics to be related, and indeed they were. For example, both strength and global integration of connectivity were positively correlated with small-worldness and clustering, whereas diversity of connections was negatively correlated with clustering. Functional networks in both groups consistently demonstrated small-world and other topological properties that have previously been described in normal human and nonhuman brain networks, and are likely to represent highly conserved principles of brain network architecture (Bassett and Bullmore, 2009; Bullmore and Sporns, 2009). Here, the shift to reduced strength and greater diversity of functional connectivity in the schizophrenic group (Fig. 6) was associated with a less clustered and hub-dominated network topology.

Some of these findings directly replicate prior fMRI and EEG reports of reduced functional connectivity, globally or regionally (Liang et al., 2006; Bluhm et al., 2007; Liu et al., 2008), or reduced clustering and small-worldness of functional networks (Micheloyannis et al., 2006; Liu et al., 2008; Rubinov et al., 2009) in schizophrenia. Notably, however, other studies have reported regionally increased functional connectivity (Zhou et al., 2007a,b; Whitfield-Gabrieli et al., 2009; Salvador et al., 2010). It is unclear why some studies should report predominantly decreased connectivity, and others increased connectivity. However, between-study differences in defining regions of interest or network nodes, differences in preprocessing strategies and connectivity metrics, and the inherent variability in small-medium sized patient samples, may all play a role. Larger and methodologically more comparable future studies will be useful.

Some of our other findings are consistent with, rather than directly replicable of, prior observations based on somewhat dif- 
ferent metrics. For example, our finding of reduced probability of high-degree hubs is compatible with previous observations of reduced degree and centrality of network hubs in schizophrenia (Rubinov et al., 2009). Likewise, our observation of greater diversity of connectivity between a single region and the rest of the brain (Fig. 3) seems compatible with prior observations of reduced homogeneity of neural activity within a single region in schizophrenia (Liu et al., 2006), given that adjacent subregions with dissimilar activity will likely show dissimilar connectivity, contributing to regional diversity. Connectivity diversity in brain networks has not been previously investigated, although studies of social interactions have used analogous metrics (Knoke and Yang, 2008). In fact, while high between-subject variability in candidate traits of schizophrenia is common (Preston and Weinberger, 2005), previous reports of any disorder-related differences in within-subject variability in fMRI are few (Manoach et al., 2001; Barch et al., 2003; Jafri et al., 2008).

Broadly speaking, many of our results are compatible with the idea that there is a "subtle randomization" of the functional network architecture in schizophrenia (Rubinov et al., 2009). Given that similar shifts to randomness, or de-differentiation, have been described as characteristic of network architectural changes with normal aging (Cabeza, 2001) and in a wide range of other disorders [including brain tumors, epilepsy, and Alzheimer's disease (Stam et al., 2009)], we need to understand more clearly which aspects of a less centralized, more robust network configuration are specific to schizophrenia and which might be common to a group of clinically distinct randomized or de-differentiated network syndromes.

\section{Relationship to other aspects of schizophrenia}

We now more speculatively consider how our analytical metrics might relate to the behavioral phenotype in schizophrenia. In this study, each subject's performance on a verbal fluency task was correlated with many of the analytical metrics. Verbal fluency tasks test the ability to generate multiple words with a given starting letter, or in a given semantic category, in limited time. Verbal fluency performance in schizophrenia has been shown to predict functional outcomes for independent living (Jaeger et al., 2003) and daily problem-solving skills (Rempfer et al., 2003; Revheim et al., 2006). In schizophrenia, verbal fluency is best predicted by psychomotor speed, rather than executive functioning or memory (van Beilen et al., 2004), and processing speed seems to mediate the link between verbal fluency performance and functional outcome in schizophrenia (Ojeda et al., 2008). Our results suggest that impaired verbal

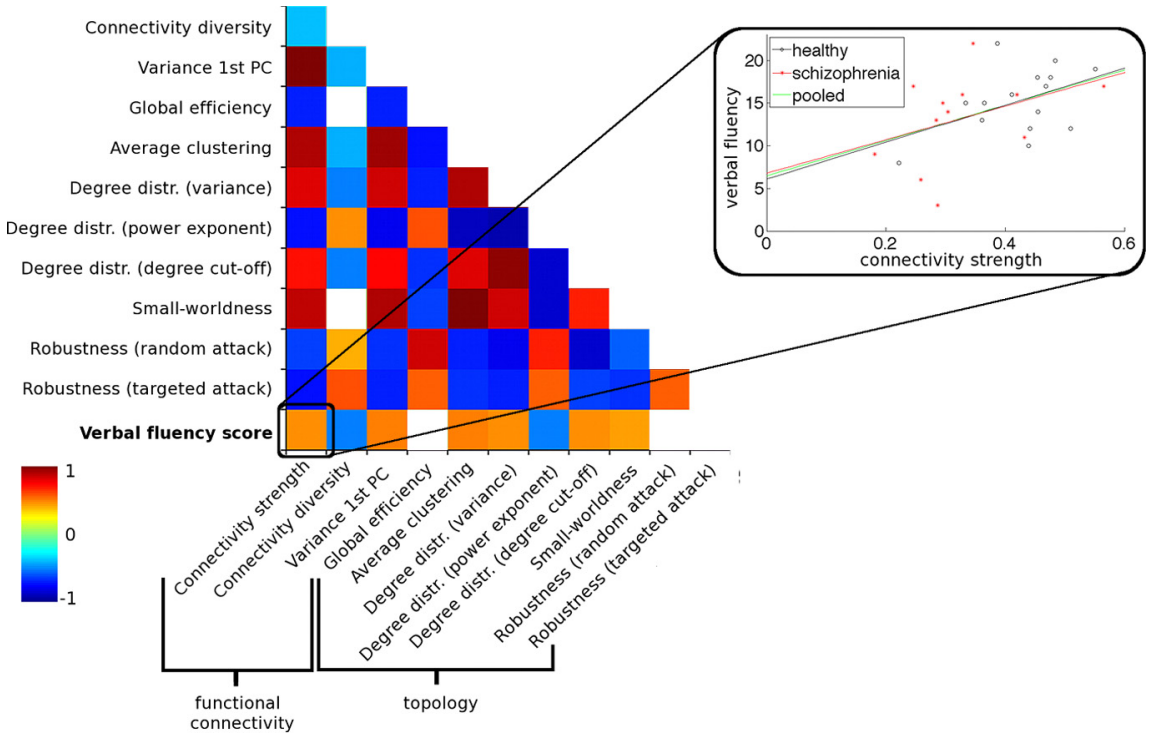

Figure 5. Matrix of correlations between global functional connectivity metrics, topological metrics, and verbal fluency score across all participants. Nonsignificant correlations $(p>0.05$ ) are left blank. The inset shows a scatter plot of verbal fluency versus connectivity strength, where the lines indicate the best linear fits for the data within each group (red, people with schizophrenia; black, healthy volunteers) and for the data pooled over both groups (green). For details, see supplemental Table 3 (available at www.jneurosci.org as supplemental material).

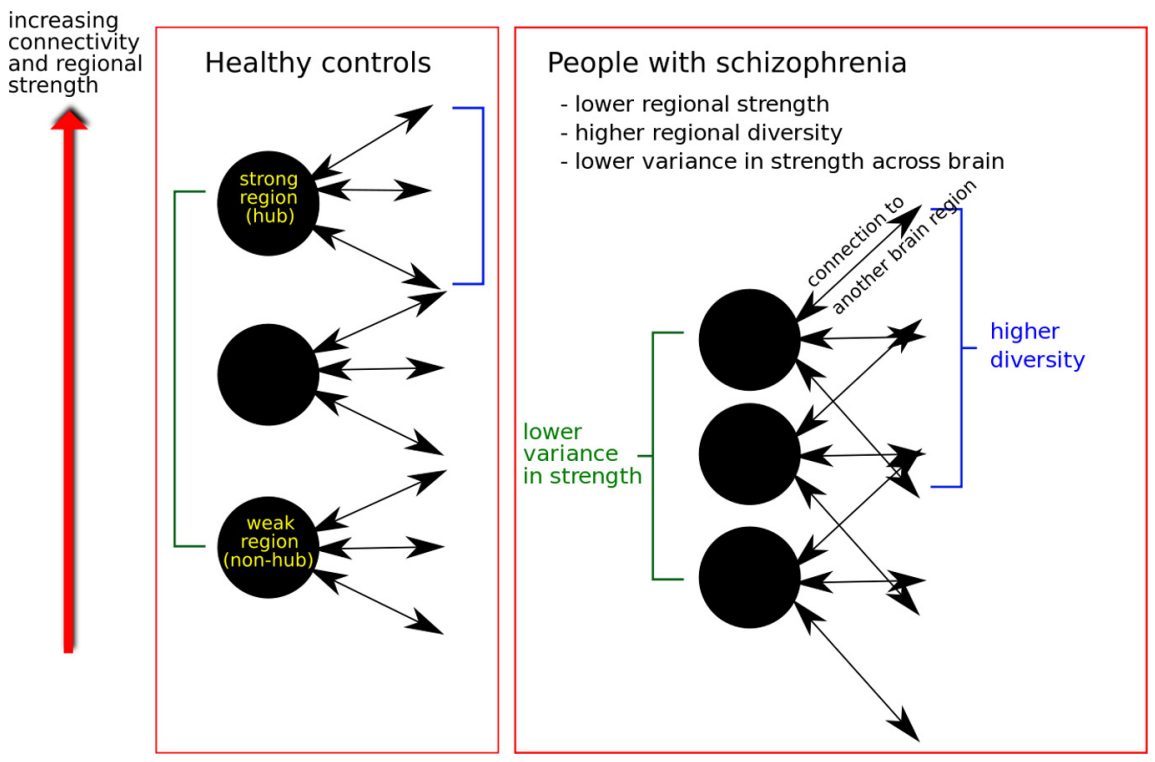

Figure 6. Hypothetical schematic of group differences in functional connectivity. People with schizophrenia show both higher diversity at each region and lower variance in connectivity strength across the brain. This can be conceptualized as a randomization or de-differentiation of functional connectivity.

fluency, presumably reflecting slower processing speed, is associated with a less strongly connected, less globally integrated, less clustered, and less hub-dominated brain functional organization. However, we did not find a significant association between task performance and global network efficiency, despite prior data and theory indicating that topological efficiency and cost efficiency correlate with intelligence and executive task performance (Dehaene and Naccache, 2001; Bassett et al., 2009; Li et al., 2009; van den Heuvel et al., 2009).

Prior evidence suggests that low-frequency functional networks are constrained by the topology of underlying anatomical networks (Honey et al., 2007). Thus we would expect the 
functional dysconnectivity of schizophrenia to be at least partly explicable in terms of anatomical disconnection, as reported in MRI or DTI studies of white matter anatomy (Bassett et al., 2008; Zhou et al., 2008); but this remains to be compellingly demonstrated. Future studies could also further test the contrasting mechanistic hypothesis that functional dysconnectivity and related network metrics are attributable to underlying abnormalities of synaptic plasticity in schizophrenia (Stephan et al., 2009).

These results, and most prior studies, have tended to focus on intuitively or demonstrably disadvantageous aspects of the schizophrenia connectome-such as lower integration of connectivity (Liu et al., 2006), lower clustering and small-worldness (Liu et al., 2008), reduced hierarchy and inefficiently increased wiring distance (Bassett et al., 2008), or reduced cost efficiency (Bassett et al., 2009). Given the high heritability of schizophrenia, and the theoretically predicted frequency of risk genes in the general population, might there be aspects of the schizophrenia connectome that confer advantages, if expressed less extremely? This could help explain persistence of risk genes despite selection pressures acting against the adverse aspects of the schizophrenia connectome.

One possible advantage identified here is the greater robustness to random attack of functional networks in schizophrenia. This means simply that the whole brain network is less likely to fragment into disconnected islands as regional nodes are removed at random. This could conceivably offer the survival advantage of greater resilience of global brain function in the face of multifocal brain lesions due to disease or injury. We might predict decreased incidence or severity of distributed brain disorders such as Alzheimer's disease in first-degree relatives of people with schizophrenia. We know of no prior data that can immediately test this hypothesis directly; but it seems intuitively convergent with prior theory that risk for Alzheimer's disease may be relatively increased in individuals with greater capacity for higher brain functions (Arendt, 2001). In any case, hub-dominated networks are less robust to random attack (Fig. 5); thus, disadvantages to integrated workspace functions due to reduced hub dominance will generally be offset by greater network robustness.

\section{Methodological limitations}

The main limitation of the study is the modest sample size $(N=$ 27), limiting the power of comparative analysis between groups and justifying a multiple-comparisons correction that effected less than maximal strength of type 1 error control at the regional level of analysis. The modest sample size, especially in relation to the number of variables considered (13), also impacted adversely on the capacity of this dataset to elucidate bivariate or multivariate associations between variables. The long acquisition time of the datasets ( $17 \mathrm{~min}$ ) will have benefited the precision of estimation of correlations and networks derived from them (Achard et al., 2008). Conversely, longer time series are less likely to represent a stable brain functional state; future studies might profitably measure behavioral arousal prospectively and/or model nonstationary or time-resolved changes in functional connectivity over the course of the scanning period (Chang and Glover, 2010) (supplemental Fig. 3, Table 9, available at www.jneurosci.org as supplemental material). Head motion may confound fMRI data, but this was individually corrected, and realignment parameters showed no between-group differences. Cardiac and respiratory sources can also contribute to variance in fMRI series, but neuronal sources are usually regarded as making the major contribution to oscillations in the frequency interval (0.06$0.125 \mathrm{~Hz}$ ) investigated here. Medication is another possible confound; dopamine receptor antagonists can alter functional connectivity and network parameters (Honey et al., 2003; Achard and Bullmore, 2007). Although people with schizophrenia were withdrawn from medication $>20$ h before scanning, mitigating acute pharmacological effects, all had been treated with antipsychotics for several years. However, antipsychotic dosage (in chlorpromazine equivalents) was not significantly correlated with any of the connectivity or network metrics. Data were scrutinized for acceptable image quality and brain regions where susceptibility artifact or incomplete brain coverage had compromised image quality in $>50 \%$ of participants were excluded: analysis was thus based on a subset of 72 regions (rather than the 90 template regions); a list of excluded regions, including inferior temporal and prefrontal regions relevant to schizophrenia, is provided in supplemental Tables 1 and 2 (available at www.jneurosci.org as supplemental material).

\section{References}

Achard S (2007) Brainwaver. Software package. Retrieved on November 30, 2009 from http://cran.r-project.org/.

Achard S, Bullmore E (2007) Efficiency and cost of economical brain functional networks. PLoS Comput Biol 3:e17.

Achard S, Salvador R, Whitcher B, Suckling J, Bullmore E (2006) A resilient, low-frequency, small-world human brain functional network with highly connected association cortical hubs. J Neurosci 26:63-72.

Achard S, Bassett DS, Meyer-Lindenberg A, Bullmore E (2008) Fractal connectivity of long-memory networks. Phys Rev E Stat Nonlin Soft Matter Phys 77:036104.

American Psychiatric Association (2000) Diagnostic and statistical manual of mental disorders, Ed 4, text revision. Washington, DC: American Psychiatric Association.

Arendt T (2001) Alzheimer's disease as a disorder of mechanisms underlying structural brain self-organization. Neuroscience 102:723-765.

Barch DM, Mathews JR, Buckner RL, Maccotta L, Csernansky JG, Snyder AZ (2003) Hemodynamic responses in visual, motor, and somatosensory cortices in schizophrenia. Neuroimage 20:1884-1893.

Bassett DS, Bullmore ET (2009) Human brain networks in health and disease. Curr Opin Neurol 22:340-347.

Bassett DS, Bullmore E, Verchinski BA, Mattay VS, Weinberger DR, MeyerLindenberg A (2008) Hierarchical organization of human cortical networks in health and schizophrenia. J Neurosci 28:9239-9248.

Bassett DS, Bullmore ET, Meyer-Lindenberg A, Apud JA, Weinberger DR, Coppola R (2009) Cognitive fitness of cost-efficient brain functional networks. Proc Natl Acad Sci U S A 106:11747-11752.

Bazire S (2005) Psychotropic drug directory. Salisbury, UK: Fivepin.

Benton A, Hamsher K, Sivan A (1976) Multilingual aphasia examination. Iowa City, IA: AJA.

Bluhm RL, Miller J, Lanius RA, Osuch EA, Boksman K, Neufeld RW, Théberge J, Schaefer B, Williamson P (2007) Spontaneous lowfrequency fluctuations in the BOLD signal in schizophrenic patients: anomalies in the default network. Schizophr Bull 33:1004-1012.

Bullmore E, Sporns O (2009) Complex brain networks: graph theoretical analysis of structural and functional systems. Nat Rev Neurosci 10:186-198.

Bullmore ET, Frangou S, Murray RM (1997) The dysplastic net hypothesis: an integration of developmental and dysconnectivity theories of schizophrenia. Schizophr Res 28:143-156.

Cabeza R (2001) Cognitive neuroscience of aging: contributions of functional neuroimaging. Scand J Psychol 42:277-286.

Campbell KE, Marsden PV, Hurlbert JS (1986) Social resources and socioeconomic status. Soc Networks 8:97-117.

Catani M, ffytche DH (2005) The rises and falls of disconnection syndromes. Brain 128:2224-2239. 
Catani M, Mesulam M (2008) What is a disconnection syndrome? Cortex 44:911-913.

Chang C, Glover GH (2010) Time-frequency dynamics of resting-state brain connectivity measured with fMRI. Neuroimage 50:81-98.

Dehaene S, Naccache L (2001) Towards a cognitive neuroscience of consciousness: basic evidence and a workspace framework. Cognition 79:1-37.

Ellison-Wright I, Bullmore E (2009) Meta-analysis of diffusion tensor imaging studies in schizophrenia. Schizophr Res 108:3-10.

Ellison-Wright I, Glahn DC, Laird AR, Thelen SM, Bullmore E (2008) The anatomy of first-episode and chronic schizophrenia: an anatomical likelihood estimation meta-analysis. Am J Psychiatry 165: 1015-1023.

Fornito A, Bullmore ET (2010) What can spontaneous fluctuations of the blood oxygenation-level-dependent signal tell us about psychiatric disorders? Curr Opin Psychiatry 23:239-249.

Friston KJ (1994) Functional and effective connectivity in neuroimaging: a synthesis. Hum Brain Mapp 2:56-78.

Friston KJ (1996) Theoretical neurobiology and schizophrenia. Br Med Bull 52:644-655.

Friston KJ, Frith CD (1995) Schizophrenia: a disconnection syndrome? Clin Neurosci 3:89-97.

Glahn DC, Laird AR, Ellison-Wright I, Thelen SM, Robinson JL, Lancaster JL, Bullmore E, Fox PT (2008) Meta-analysis of gray matter anomalies in schizophrenia: application of anatomic likelihood estimation and network analysis. Biol Psychiatry 64:774-781.

Honey CJ, Kötter R, Breakspear M, Sporns O (2007) Network structure of cerebral cortex shapes functional connectivity on multiple time scales. Proc Natl Acad Sci U S A 104:10240-10245.

Honey GD, Suckling J, Zelaya F, Long C, Routledge C, Jackson S, Ng V, Fletcher PC, Williams SCR, Brown J, Bullmore ET (2003) Dopaminergic drug effects on physiological connectivity in a human cortico-striatothalamic system. Brain 126:1767-1781.

Humphries MD, Gurney K, Prescott TJ (2006) The brainstem reticular formation is a small-world, not scale-free, network. Proc Biol Sci 273:503-511.

Jaeger J, Czobor P, Berns SM (2003) Basic neuropsychological dimensions in schizophrenia. Schizophr Res 65:105-116.

Jafri MJ, Pearlson GD, Stevens M, Calhoun VD (2008) A method for functional network connectivity among spatially independent resting-state components in schizophrenia. Neuroimage 39:1666-1681.

Kaiser M, Hilgetag CC (2006) Nonoptimal component placement, but short processing paths, due to long-distance projections in neural systems. PLoS Comput Biol 2:e95.

Kay SR, Fiszbein A, Opler LA (1987) The Positive and Negative Syndrome Scale (PANSS) for schizophrenia. Schizophr Bull 13:261-276.

Knoke D, Yang S (2008) Social network analysis. Newberry Park, CA: Sage.

Latora V, Marchiori M (2001) Efficient behavior of small-world networks. Phys Rev Lett 87:198701.

Li Y, Liu Y, Li J, Qin W, Li K, Yu C, Jiang T (2009) Brain anatomical network and intelligence. PLoS Comput Biol 5:e1000395.

Liang M, Zhou Y, Jiang T, Liu Z, Tian L, Liu H, Hao Y (2006) Widespread functional disconnectivity in schizophrenia with resting-state functional magnetic resonance imaging. Neuroreport 17:209-213.

Liu H, Liu Z, Liang M, Hao Y, Tan L, Kuang F, Yi Y, Xu L, Jiang T (2006) Decreased regional homogeneity in schizophrenia: a resting state functional magnetic resonance imaging study. Neuroreport 17:19-22.

Liu Y, Liang M, Zhou Y, He Y, Hao Y, Song M, Yu C, Liu H, Liu Z, Jiang T (2008) Disrupted small-world networks in schizophrenia. Brain 131: 945-961.

Manoach DS, Halpern EF, Kramer TS, Chang Y, Goff DC, Rauch SL, Kennedy DN, Gollub RL (2001) Test-retest reliability of a functional MRI working memory paradigm in normal and schizophrenic subjects. Am J Psychiatry 158:955-958.

Meunier D, Achard S, Morcom A, Bullmore E (2009) Age-related changes in modular organization of human brain functional networks. Neuroimage 44:715-723.

Meyer-Lindenberg A, Weinberger DR (2006) Intermediate phenotypes and genetic mechanisms of psychiatric disorders. Nat Rev Neurosci 7:818-827.

Micheloyannis S, Pachou E, Stam CJ, Breakspear M, Bitsios P, Vourkas M,
Erimaki S, Zervakis M (2006) Small-world networks and disturbed functional connectivity in schizophrenia. Schizophr Res 87:60-66.

Minzenberg MJ, Laird AR, Thelen S, Carter CS, Glahn DC (2009) Metaanalysis of 41 functional neuroimaging studies of executive function in schizophrenia. Arch Gen Psychiatry 66:811-822.

Nelson H (1992) National Adult Reading Test (NART): test manual. Windsor, UK: National Foundation for Educational Research.

Ojeda N, Peña J, Sánchez P, Elizagárate E, Ezcurra J (2008) Processing speed mediates the relationship between verbal memory, verbal fluency, and functional outcome in chronic schizophrenia. Schizophr Res 101:225-233.

Percival D, Walden A (2000) Wavelet methods for time series analysis. Cambridge, UK: Cambridge UP.

Preston GA, Weinberger DR (2005) Intermediate phenotypes in schizophrenia: a selective review. Dialogues Clin Neurosci 7:165-179.

Rempfer MV, Hamera EK, Brown CE, Cromwell RL (2003) The relations between cognition and the independent living skill of shopping in people with schizophrenia. Psychiatry Res 117:103-112.

Revheim N, Schechter I, Kim D, Silipo G, Allingham B, Butler P, Javitt DC (2006) Neurocognitive and symptom correlates of daily problem-solving skills in schizophrenia. Schizophr Res 83:237-245.

Rubinov M, Knock SA, Stam CJ, Micheloyannis S, Harris AW, Williams LM, Breakspear M (2009) Small-world properties of nonlinear brain activity in schizophrenia. Hum Brain Mapp 30:403-416.

Salvador R, Sarró S, Gomar JJ, Ortiz-Gil J, Vila F, Capdevila A, Bullmore E, McKenna PJ, Pomarol-Clotet E (2010) Overall brain connectivity maps show cortico-subcortical abnormalities in schizophrenia. Hum Brain Mapp. Advance online publication. Retrieved June 26, 2010. doi:10.1002/hbm.20993.

Schank T, Wagner D (2005) Approximating clustering coefficient and transitivity. J Graph Algorithms Appl 9:265-275.

Sheehan DV, Lecrubier Y, Sheehan KH, Amorim P, Janavs J, Weiller E, Hergueta T, Baker R, Dunbar GC (1998) The Mini-International Neuropsychiatric Interview (M.I.N.I.): the development and validation of a structured diagnostic psychiatric interview for DSM-IV and ICD-10. J Clin Psychiatry 59 [Suppl 20]:22-33.

Stam CJ, de Haan W, Daffertshofer A, Jones BF, Manshanden I, van Cappellen van Walsum AM, Montez T, Verbunt JP, de Munck JC, van Dijk BW, Berendse HW, Scheltens P (2009) Graph theoretical analysis of magnetoencephalographic functional connectivity in Alzheimer's disease. Brain 132:213-224.

Stephan KE, Friston KJ, Frith CD (2009) Dysconnection in schizophrenia: from abnormal synaptic plasticity to failures of self-monitoring. Schizophr Bull 35:509-527.

Suckling J, Long C, Triantafyllou C, Brammer M, Bullmore E (2006) Variable precision registration via wavelets: optimal spatial scales for intersubject registration of functional MRI. Neuroimage 31:197-208.

Tononi G, Sporns O, Edelman GM (1994) A measure for brain complexity: relating functional segregation and integration in the nervous system. Proc Natl Acad Sci U S A 91:5033-5037.

Tzourio-Mazoyer N, Landeau B, Papathanassiou D, Crivello F, Etard O, Delcroix N, Mazoyer B, Joliot M (2002) Automated anatomical labeling of activations in SPM using a macroscopic anatomical parcellation of the MNI MRI single-subject brain. Neuroimage 15:273-289.

van Beilen M, Pijnenborg M, van Zomeren EH, van den Bosch RJ, Withaar FK, Bouma A (2004) What is measured by verbal fluency tests in schizophrenia? Schizophr Res 69:267-276.

van den Heuvel MP, Stam CJ, Kahn RS, Hulshoff Pol HE (2009) Efficiency of functional brain networks and intellectual performance. J Neurosci 29:7619-7624.

Van Essen DC (2005) A population-average, landmark- and surface-based (PALS) atlas of human cerebral cortex. Neuroimage 28:635-662.

Van Essen DC, Drury HA, Dickson J, Harwell J, Hanlon D, Anderson CH (2001) An integrated software suite for surface-based analyses of cerebral cortex. J Am Med Inform Assoc 8:443-459.

Volkow ND, Wolf AP, Brodie JD, Cancro R, Overall JE, Rhoades H, van Gelder P (1988) Brain interactions in chronic schizophrenics under resting and activation conditions. Schizophr Res 1:47-53.

Watts DJ, Strogatz SH (1998) Collective dynamics of 'small-world' networks. Nature 393:440-442. 
Wechsler D (1997) The Wechsler memory scale-III, UK manual. San Antonio, TX: Psychological Corporation.

Weinberger DR, Berman KF, Suddath R, Torrey EF (1992) Evidence of dysfunction of a prefrontal-limbic network in schizophrenia: a magnetic resonance imaging and regional cerebral blood flow study of discordant monozygotic twins. Am J Psychiatry 149:890-897.

Whitfield-Gabrieli S, Thermenos HW, Milanovic S, Tsuang MT, Faraone SV, McCarley RW, Shenton ME, Green AI, Nieto-Castanon A, LaViolette P, Wojcik J, Gabrieli JD, Seidman LJ (2009) Hyperactivity and hyperconnectivity of the default network in schizophrenia and in first-degree relatives of persons with schizophrenia. Proc Natl Acad Sci U S A 106: $1279-1284$
Woods SW (2003) Chlorpromazine equivalent doses for the newer atypical antipsychotics. J Clin Psychiatry 64:663-667.

Zhou Y, Liang M, Jiang T, Tian L, Liu Y, Liu Z, Liu H, Kuang F (2007a) Functional dysconnectivity of the dorsolateral prefrontal cortex in first-episode schizophrenia using resting-state fMRI. Neurosci Lett 417:297-302.

Zhou Y, Liang M, Tian L, Wang K, Hao Y, Liu H, Liu Z, Jiang T (2007b) Functional disintegration in paranoid schizophrenia using resting-state fMRI. Schizophr Res 97:194-205.

Zhou Y, Shu N, Liu Y, Song M, Hao Y, Liu H, Yu C, Liu Z, Jiang T (2008) Altered resting-state functional connectivity and anatomical connectivity of hippocampus in schizophrenia. Schizophr Res 100:120-132. 